\section{Minor head injury in anticoagu- lated patients: a 6-year retrospective analysis in an emergency department}

\author{
Alessandro Riccardi, Grazia Guiddo, \\ Bruno Chiarbonello, Flavio Frumento, \\ Piera Polletti, Marina Castelli, \\ Pierangela Minuto, Roberto Lerza \\ Emergency Department, San Paolo \\ Hospital, Savona, Italy
}

\section{Abstract}

The appropriate management of minor head injury (MHI) in patients receiving oral anticoagulant $(\mathrm{OAC})$ is unclear. In this retrospective study, we focused on elderly patients $(>65$ years) treated with $\mathrm{OAC}$, presented to our emergency department with MHI between 2004 and 2010. Three hundred and six patients with MHI were taking OAC: we documented $7.19 \%$ hemorrhages at the first computed tomography (C); $18.19 \%$ deaths; $50.1 \%$ spontaneous reabsorptions; $22.73 \%$ deteriorations of intracranial bleeding without surgical intervention (for clinical comorbidity), and $4.55 \%$ neurosurgical interventions. We documented a second positive CT scan in 2 patients (1.51\%) who had no symptoms and remained asymptomatic during observation. In both cases, intracranial bleeding resolved spontaneously. The mean international normalized ratio (INR) value was 2.26, higher in the group of patients with bleeding (2.74) than in the group without bleeding (2.19). We found a significant increased risk in patients with posttraumatic loss of consciousness [odds ratio (OR) 28.3], diffuse headache (OR 14.79), vomiting (OR 14.2) and neurological signs (OR 5.27). We did not reach significance in patients with post-traumatic amnesia. Our data confirm the need for a CT scan of any patients on OAC with MHI. None of our patients developed any symptoms or signs during observation, and only 2 patients developed an intracranial hemorrhage in the second CT scan with a favorable evolution. Our data need to be confirmed with an observational study, but we suggest that the second CT could be reserved for patients developing symptoms and signs during observation. We also underline the role of the INR in the stratification of risk.

\section{Introduction}

Minor head injury (MHI) in patients receiving oral anticoagulant (OAC) is a real problem, and the appropriate management of these patients is still unclear, ${ }^{1-4}$ probably due to the lack of substantial evidence. The issue is particularly marked in elderly patients: an increasing number of individuals are receiving anticoagulant medication for various conditions such as atrial fibrillation, valvular disease and thromboembolic disease, which are more prevalent in the aging population. ${ }^{5-7}$ The European Society of Cardiology Guidelines for management of atrial fibrillation, clearly indicate the need of OACs for a great number of patients, and for any patients older than 75 years. ${ }^{8}$ Furthermore, the elderly are more prone to hemorrhagic injuries, especially those associated with falls. ${ }^{6} \mathrm{~A}$ small number of studies regarding the effects of preinjury anticoagulation in elderly trauma patients is available in the literature, and their conclusions are often contradictory. ${ }^{2-6,9}$

Several guidelines and rules had been produced for focusing on the main management problems of minor head injury, such as the period of observation of the patient, the right time for discharge, assessments and specialized equipment needed. ${ }^{1,2,10-13}$ Long-term oral anticoagulation has been identified as a significant risk factor for intracranial injury in minor head injury, and computed tomography (CT) scanning is generally recommended for such patients regardless of clinical presentation. ${ }^{2,3,6}$ Some authors and also UK's National Institute for Health and Care Excellence guidelines disagree with this statement. ${ }^{1}$ A major point of discussion is the further management of these patients, and the repetition of head CT scans before discharge. ${ }^{5-7}$ The problem is that once bleeding occurs, the reported mortality varies from 20 to $50 \%,{ }^{7}$ and it is important to identify useful protocols in order to minimize this risk. According to Italian Guidelines, all anticoagulated patients with minor head injury receive an initial CT scan, admission for a 24-h period of close neurologic observation in an observation unit, and then a second CT scan before discharge. ${ }^{2}$

In this retrospective study, we focused on elderly patients ( $>65$ years) treated with OACs, presented to our emergency medicine department with minor head injury, with the aim of evaluating the risk of development of intracranial complications in these patients.

\section{Materials and Methods}

We performed a retrospective study by retrieving and reviewing patients over 65 years old evaluated in our emergency department (ED) (San Paolo Hospital, Savona, Italy) in which CT scans of the head were performed for minor head injury, between April 2004 and April 2010. All cranial CT scans were 32 slice
Correspondence: Alessandro Riccardi, Emergency Department, San Paolo Hospital, via Genova, 17100 Savona, Italy.

Tel. +39.019 .8404963 - Fax: +39.019 .8404529 .

E-mail: a.riccardi@asl2.liguria.it; dottriccardi@ yahoo.it

Key words: Minor head injury, oral anticoagulant, elderly.

Conflict of interests: the authors declare no potential conflict of interests.

Contributions: AR, study design and statistical analysis performance; $\mathrm{GG}$, data collection and cowriting of the paper; $\mathrm{BC}$, data collection, co-writing of the paper and statistical analysis performance; PP, literature review, references collection and co-writing of the paper; MC, data collection, co-writing of the paper and tables-figures preparation; PM, data collection, co-writing of the paper and to references reviewing; RL, study design, co-writing of the paper and whole work conduction.

Received for publication: 7 September 2013. Revision received: 25 November 2013.

Accepted for publication: 20 December 2013.

This work is licensed under a Creative Commons Attribution 3.0 License (by-nc 3.0).

(C) Copyright A. Riccardi et al., 2014 Licensee PAGEPress, Italy

Emergency Care Journal 2014; 10:1913

doi:10.4081/ecj.2014.1913

scans without contrast.

We defined minor head injury patients with GCS of 14-15, according to Italian Guidelines ${ }^{2}$ and according to the criteria adopted in our previous study. ${ }^{14}$

We analyzed the first CT scans of the head, and if executed, the second CT prior to discharging. In case of pathological findings correlated to trauma, we documented the further treatment of these patients.

Any variations of neurological examinations, the development of symptoms like vomiting, diffuse headache and loss of consciousness during the observation were recorded. We also recorded some demographic factors such as age, sex, the indication for the anticoagulant, the type of anticoagulant prescribed, the symptoms and GCS at time of presentation, the dynamics of trauma and international normalized ratio (INR) value. Our primary outcome was any intracranial traumatic findings on CT; a secondary outcome was any neurosurgical intervention, death or any deterioration. We defined a neurosurgical intervention as any invasive procedure performed for treatment of complications correlated to head injury. 


\section{Data analysis}

Descriptive statistics for study populations were calculated. We also calculated the odds ratio (OR) for acute findings on CT in patients on OAC and low risk minor head injury (according to Italian guidelines): we defined low risk MHI patients with GCS 14-15, a clear dynamics of trauma with exclusion of dangerous event, no or minor wounds, absence of neurological defects or history of neurological disease or previous neurosurgical intervention, no symptoms (headache, loss of consciousness or vomiting) except pain at the site of the injury

We also calculated statistics in the group of patients without bleeding in the first or second CT scan, compared with patient with bleeding.

We performed statistical analysis [interval of confidence (IC) $96 \%$, standard deviation (SD), OR] using an electronic spreadsheet.

\section{Results and Discussion}

Between April 2004 and April 2010 we evaluated 4518 patients over 65 years (65-107 years) for head injury. Among these, 306 patients were taking anticoagulants (warfarin) and were eligible for the study, with a mean age of 80.34 years: in this group of patients we documented 22 intracranial hemorrhages at the first CT on admission (7.19\%) (Table 1). All patients had a normal platelet count.

Among 306 anticoagulated patients, we considered the 246 patients with low risk of minor head injury (see Methods) for treatment with anticoagulant, in order to isolate just the OAC as a risk factor. In this group, we documented 14 bleeding episodes (5.70\%), 12 in the first CT scan and 2 in the second CT after observa- tion (with first negative CT). Compared to the 2149 patients at low risk of minor head injury and age $>65$ years, but no anticoagulant, the OR of intracranial bleeding was 2.82 $(\mathrm{P}<0.001)$.

In 152 patients, second CTs were not executed, based on the patients' wishes, normal
INR at first evaluation or subtherapeutical range, or for in-hospital admission for other associated reasons (fractures, internal or surgical diseases). In the first negative CT scan of patients admitted to our observational unit (132 patients), we documented a second positive CT scan in 2 patients (1.51\%): these

Table 1. Intracranial bleeding in head injured patients taking anticoagulants classified according to age.

\begin{tabular}{lccccc} 
& n & Mean age & SD & IC 95\% & P \\
Overall & 306 & 80.34 & 6.0545 & $79.653-81.018$ & - \\
Patients with positive findings on CT & 22 & 79.84 & 5.929554 & $77.37-82.32$ & 0.69 \\
\hline Patients with negative CT scan of the head & 284 & 80.37 & 6.072679 & $79.66-81.08$ & 0.69 \\
\hline
\end{tabular}

SD, standard deviation; IC, interval of confidence; CT, computed tomography.

Table 2. Intracranial bleeding in head injured patients taking anticoagulants classified according to mean international normalized ratio.

\begin{tabular}{lccccc} 
& n & Mean INR & SD & IC 95\% & P \\
Overall & 306 & 2.25 & 0.817 & $2.14-2.35$ & - \\
Patients with positive findings on CT & 22 & 2.74 & 0.582 & $2.49-2.99$ & $<0.05$ \\
\hline Patients with negative CT scan of the head & 284 & 2.19 & 0.82 & $2.08-2.31$ & $<0.05$ \\
\hline
\end{tabular}

INR, international normalized ratio; SD, standard deviation; IC, interval of confidence; CT, computed tomography.

Table 3. Risk of intracranial bleeding according to the presence of post traumatic symptoms.

\begin{tabular}{lccccc} 
Post traumatic symptoms & Patients (n) & $\begin{array}{r}\text { Intracranial bleedings } \\
\text { n }\end{array}$ & OR & P \\
Loss of consciousness & 3 & 2 & 66.67 & 28.3 & $<0.001$ \\
Diffuse headache & 6 & 3 & 50 & 14.79 & $<0.001$ \\
\hline Vomiting & 4 & 2 & 50 & 14.2 & $<0.005$ \\
Neurological defects & 20 & 5 & 25 & 5.27 & $<0.005$ \\
\hline Amnesia & 9 & 1 & 11.12 & 1.64 & 0.64 \\
\hline OR, odds ratio & & & & &
\end{tabular}

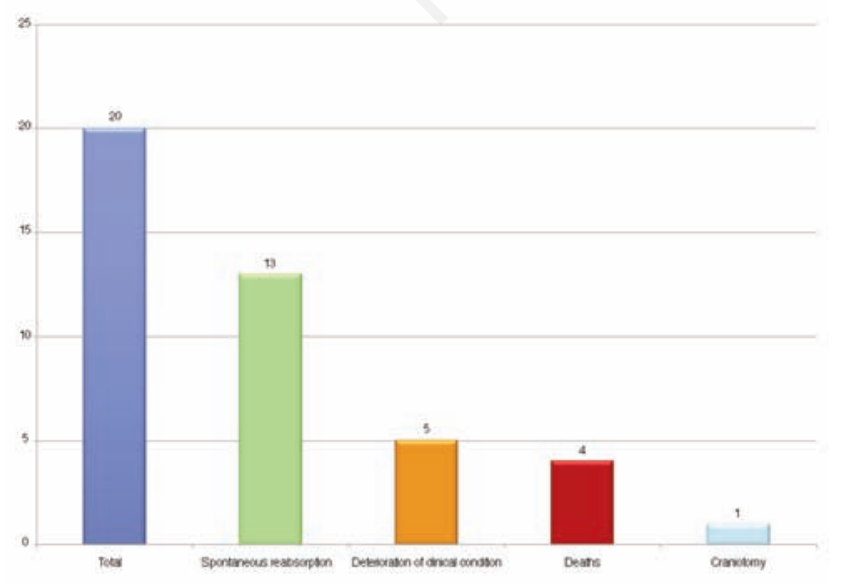

Figure 1. Outcomes of patients with initial positive computed tomography.

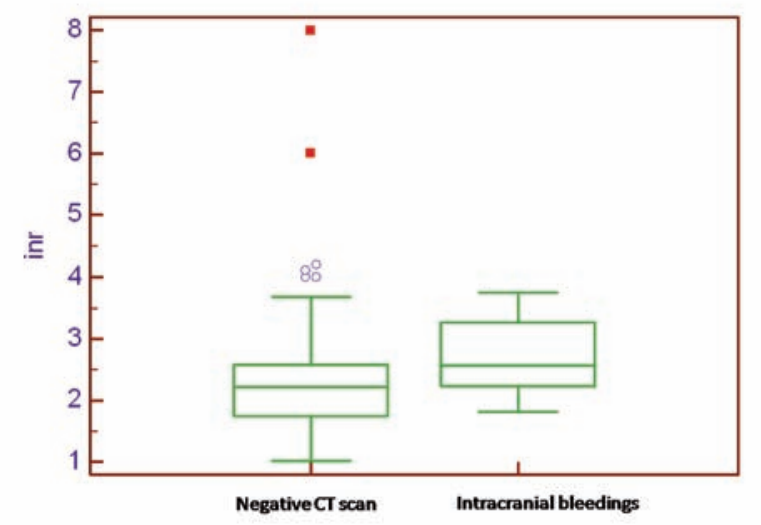

Figure 2. Mean international normalized ratio in head injured patients taking anticoagulants. 
patients had no symptoms at first evaluation, and remained asymptomatic during observation. In both cases, intracranial bleeding (1 subarachnoideal bleeding and 1 subdural hematoma) resolved spontaneously in a few days, and no surgical interventions were required.

In the 20 patients with initial positive CT scan from the beginning (3 subdural hematoma, 7 subarachnoideal hemorrhage and 10 intraparenchimal bleeding episodes), we documented 4 deaths (18.19\% of bleeding, and $1.30 \%$ of overall patients); in 13 patients we documented spontaneous reabsorption of bleeding (50.1 and 4.25\%); in five patients we documented a deterioration of intracranial bleeding, without surgical intervention (for clinical comorbidity) (22.73 and 1.63\%), and 1 patient (4.55 and 0.33\%) underwent neurosurgical intervention (craniotomy) (Figure 1). The mean INR value was 2.2576 . In the group of patients with bleeding (22 patients) the mean INR was 2.74, and in the group of patients without bleeding the mean INR was 2.19 , with a significant T-students test and Kruskal Wallis test $(\mathrm{P}<0.005)$ (Table 2, Figure $2)$. We analyzed the risk of intracranial bleeding according to the presence of symptoms (Table 3): we found a significant increased risk in patients with post traumatic loss of consciousness (OR 28.3, $\mathrm{P}<0.001)$, diffuse headache (OR 14.79, $\mathrm{P}<0.001$ ), vomiting (OR 14.2, $\mathrm{P}<0.005)$ and neurological signs ( $\mathrm{OR}$ $5.27, \mathrm{P}<0.005)$. We did not reach any significance in patients with post traumatic amnesia. These data are based on small number of patients: among the 60 patients excluded from the overall population of 306 patients (because not responding to Italian Guidelines Criteria), we documented 42 patients presenting with symptoms.

\section{Conclusions}

A review ${ }^{3}$ indicated that there is no agreement about the proper use and timing of CT scan of the head in trauma patients on anticoagulant treatment. ${ }^{4,15}$ Taking OAC is considered a risk factor for developing intracranial hemorrhage after minor head injury: ${ }^{6}$ our data confirm this statement, and demonstrate a strong increase in the rate of intracranial hemorrhage also in patients without other risk factors $\mathbf{7 . 1 9 \%}$ of intracranial complications in the overall anticoagulated patients, and 5.70\% in the low risk group); this is particularly relevant in comparison with the percentage of complications in non-anticoagulated patients. So, as indicated in the European Guidelines, ${ }^{16}$ our data confirm the need for a CT scan of the head of any patients on oral anticoagulation treatment with a minor head injury, even in absence of any risk factors such as symptoms or dangerous dynamics. Regarding the $24 \mathrm{~h}$ observation period and the need of a second CT of the head before discharging patients, our data appear in contrast to other authors. ${ }^{6}$ None of our patients developed any symptoms or signs during observation, and only 2 patients developed an intracranial hemorrhage in the second CT scan. Both patients were asymptomatic, and the evolution was favorable, with a spontaneous reabsorption in a few days. Our data need to be confirmed with an observational study, they lack significance because our patients were mainly at low risk, so this could introduce a bias in our analysis: in other papers, the development of complications during observation was higher, ${ }^{5-8}$ but their population presented a higher percentage of patients with other risk factors (such as headache, vomiting and loss of consciousness). So, the observation seemed to be necessary, for the identification of even rare complications, because mortality and disability in patients on anticoagulation treatment who develop complications remain high. We also suggest that the second CT could be reserved for patients developing symptoms and signs during observation.

We have to underline the role of the INR in the stratification of risk: we documented a higher value of mean INR in the group of patients with intracranial hemorrhage, in relation to patients without complications. This appears to be obvious, but it is important to consider one factor: the value of INR influenced the risk in the first CT scan, but did not increase the risk of developing intracranial hemorrhage during observation. Nevertheless it is important to suspend the oral anticoagulation in any case if it is possible or safe: on this point, however, there is no agreement in the literature..$^{3,17,18}$

Another important point to underline is the risk of late complications, like subdural hematoma, higher in the elderly ( $>80$ years old) than in younger patients. ${ }^{14}$ The increase in space between the brain and skull allows for the expansion of intracranial content, in weeks or months with fewer symptoms. ${ }^{19,20}$ This complication appears to be more significant in anticoagulated patients, ${ }^{6}$ but it is not realistic to increase the observation period above the standard $24 \mathrm{~h}$. So, for discharged patients, it is important to indicate clearly the need for urgent evaluation in case of deterioration, diffuse headache or any symptoms not directly correlated to the head trauma, because of the long period of time (sometimes months). In alternative to a second CT before discharging asymptomatic patients, it might be advisable to plan a second CT scan of the head after a longer interval, i.e. 7 days, with the aim of visualizing a greater number of complications.

\section{Limitations of the study}

The major limit of our study consisted in the health and hospital organization of our district. Our hospital (city hospital with mean ED access 55,000/year) is not a trauma center. The latter is located $20 \mathrm{~km}$ away and has a neurosurgery unit; so a number of patients with severe symptoms or with dangerous dynamics of trauma, because of their anticoagulation, were probably directly transferred (or they directly reached) to the hospital with neurosurgery, and this likely introduced a selection of our population.

\section{References}

1. NICE. Head injury triage, assessment, investigation and early management of head injury in infants, children and adults. Clinical guideline 56. London: National Institute for Clinical Excellence; 2007. Available from: http://www.nice.org.uk/nicemedia/ pdf/CG56NICEGuideline.pdf

2. Leiblich A, Mason S. Emergency management of minor head injury in anticoagulated patients. Emerg Med J 2011;28:115-8.

3. Gittleman AM, Ortiz A0, Keating DP, Katz DS. Indications for CT in patients receiving anticoagulation after head trauma. Am J Neuroradiol 2005;26:603-6.

4. Reynolds FD, Dietz PA, Higgins D, Whitaker TS. Time to deterioration of the elderly, anticoagulated, minor head injury patient who presents without evidence of neurologic abnormality. J Trauma 2003;54: 492-6.

5. Menditto VG, Lucci M, Polonara S, et al. Management of minor head injury in patients receiving oral anticoagulant therapy: a prospective study of a 24-hour observation protocol. Ann Emerg Med 2012;59:451-5.

6. Lavoie A, Ratte S, Clas D, et al. Preinjury warfarin use among elderly patients with closed head injuries in a trauma center. $\mathrm{J}$ Trauma 2004;56:802-7.

7. Kaen A, Jimenez-Roldan L, Arrese I, et al. The value of sequential computed tomography scanning in anticoagulated patients suffering from minor head injury. $\mathrm{J}$ Trauma 2010;68:895-8.

8. Camm AJ, Kirchhof P, Lip GY, et al. European Heart Rhythm AssociationEuropean Association for cardio-thoracic surgery guidelines for the management of atrial fibrillation: the task force for the management of atrial fibrillation of the European Society of Cardiology (ESC). Eur Heart J 2010;31:2369-429.

9. Mina AA, Bair HA, Howells GA, Bendick PJ. Complications of preinjury warfarin use in 
the trauma patient. J Trauma 2003;54:842 7.

10. Stiell IG, Wells GA, Vandemheen K, et al. The Canadian CT head rule for patients with minor head injury. Lancet 2001;357:1391-6.

11. Haydel MJ, Preston CA, Mills TJ, et al. Indications for computed tomography in patients with minor head injury. New Engl J Med 2000;343:100-5.

12. Smits M, Dippel DWJ, de Haan GG, et al. Minor head injury: guidelines for the use of CT - a multicenter validation study. Radiology 2007;245:831-8.

13. Ingebrigtsen T, Romner B, Kock-Jensen C. Scandinavian guidelines for initial management of minimal, mild, and moderate head injuries. J Trauma 2000;48:1985-90.

14. Riccardi A, Frumento F, Guiddo G, et al. Minor head injury in the elderly at very low risk: a retrospective study of 6 years in an Emergency Department (ED). Am J Emerg Med 2013;31:37-41.

15. Peck KA, Sise CB, Shackford SR, et al. Delayed intracranial hemorrhage after blunt trauma: are patients on preinjury anticoagulants and prescription antiplatelet agents at risk? J Trauma 2011;71:1600-4.

16. Vos PE, Battistin L, Birbamer G, et al. EFNS guideline on mild traumatic brain injury: report of an EFNS task force. Eur J Neurol 2002;9:207-19.

17. Li J, Brown J, Levine M. Mild head injury, anticoagulants and risk of intracranial injury. Lancet 2001;357:771e2.

18. Fabbri A, Vandelli A, Servadei F, Marchesini G. Coagulopathy and NICE recommendations for patients with mild head injury. J Neurol Neurosur Ps 2004;75: $1787 \mathrm{e} 8$.

19. Mack LR, Chan SB, Silva JC, Hogan TM. The use of head computed tomography in elderly patients sustaining minor head trauma. J Emerg Med 2003;24:157-62.

20. Samaras N, Chevalley T, Samaras D, Gold G. Older patients in the emergency department: a review. Ann Emerg Med 2010;56: 261-9. 